\title{
Maintenant : Claude Simon
}

Dominique Viart

\section{(2) OpenEdition}

\section{Journals}

Édition électronique

URL : http://journals.openedition.org/ccs/460

DOI : $10.4000 /$ ccs.460

ISSN : 2558-782X

\section{Éditeur :}

Presses universitaires de Rennes, Association des lecteurs de Claude Simon

\section{Édition imprimée}

Date de publication : 31 décembre 2006

Pagination : 11-14

ISBN : 9782914518895

ISSN : 1774-9425

\section{Référence électronique}

Dominique Viart, « Maintenant: Claude Simon », Cahiers Claude Simon [En ligne], 2 | 2006, mis en ligne le 20 septembre 2017, consulté le 24 septembre 2020. URL : http://journals.openedition.org/ccs/460 ; DOI : https://doi.org/10.4000/ccs.460 


\section{Maintenant: Claude Simon}

par Dominique VIART

Il avait vu la mort en face, plusieurs fois : "Maintenant. Maintenant. Maintenant... ». C'était au cours de la Seconde Guerre mondiale, ou, plus tard, en affrontant la maladie. Mais pour nous qui le lisons, c'est dans La Route des Flandres, dans L'Acacia ou dans Le Jardin des Plantes, tant le texte s'est approprié ce qui n'existe pas en dehors de lui. Et cela demeure, cependant, hors de La Route des Flandres, de L'Acacia et des autres livres, car, comme Claude Simon le dit lui même, citant Conrad : "Non, c'est impossible : il est impossible de communiquer la sensation vivante d'aucune époque donnée de son existence - ce qui fait sa vérité, son sens - sa subtile et pénétrante essence. C'est impossible. Nous vivons comme nous rêvons - seuls ". Mais ce qui demeure hors des livres, comme un point de fuite, sa phrase s'acharne à le rejoindre, attelée à cette tâche depuis l'origine. Et d'abord peut-être sans vraiment le savoir, dès les tout premiers romans. De ce dehors des mots elle fait un point de fuite en avant des livres, et non en arrière comme un souvenir ou des images déposés dans quelques replis de la mémoire, qu'il suffirait de retrouver, et de déplier. Un point de fuite en avant des textes, qui les aspire vers plus de détails, plus de précision quand bien même la précision elle-même est aléatoire, incertaine et toujours remise en question. 
Il avait vu sa mort en face, imminente. Et cela-même eût pu susciter une écriture de l'urgence, convulsée, assaillie par tout ce qu'il faut dire encore, avant qu'il ne soit trop tard. Car même si l'on en réchappe, on demeure alors, pour toujours, un survivant. Et l'on sait, désormais, que cette vie aura une fin, que tout disparaîtra aux yeux qui se fermeront. On le sait, non comme une abstraction, mais avec tout son corps, avec sa chair et ses sens, avides d'éprouver et de sentir encore, avec " cette déchirante et mélancolique avidité avec laquelle le condamné à mort regarde le monde autour de lui ». Aussi l'écriture de Claude Simon est-elle bien d'abord une écriture du corps, heurtée aux matérialités du monde et sensible à ses séductions. À sa "somptueuse magnificence ", dont le corps se pénètre sans exclusives : bruits, odeurs, images, parfums, contacts, pestilences, peintures, paysages... Tout y est matière pour les sens dans le déploiement des mots.

Une écriture de l'urgence, sans doute, par sa ténacité à revenir au papier, à ne pas lâcher l'objet qu'elle se donne. Mais pas comme on l'entend peut-être : elliptique, haletante. L'urgence, ici, est dans la saisie des choses, dans la prise verbale qu'elle s'accorde sur elles; pas dans le fragment ni dans la cavalcade. Au contraire : plutôt que de se hâter contre le temps, l'écriture de Claude Simon en exige le suspens. Et le plus fort est qu'elle l'obtient. C'est la phrase qui l'obtient. C'est le phrasé de sa phrase. Sa hâte à ne pas la finir, son souci de la relancer, d'y ouvrir des digressions, d'en multiplier les arborescences. Comme si, tant que la phrase continuait, le monde ne pouvait plus finir. Il avait vu sa mort imminente : "Maintenant. Maintenant. Maintenant...» - et il la retenait. Ou plutôt il retenait le monde au point de disparaître, peu désireux de s'en séparer, fûtil funèbre, pourrissant parfois autant que fleurissant, prolixe, redondant ou baroque. Main - tenant : il retenait le monde de la main, plume à la main, de sa fine écriture continue s'amuïssant au bord droit de la feuille.

Le monde : présent comme une énigme, «impénétrable » - c'est son mot - indifférent à se qui se trame en lui, mais d'une présence inégalable, que sa phrase pourtant s'efforçait d'égaler - et qu'elle égalait, égarant son lecteur dans ses méandres, le capturant dans ses rets, ses 
dérives, ses départs et ses retours, ses parenthèses ouvertes sur d'autres mondes encore dans le monde, hypothèses et suppositions, analogies, comparaisons, suspicions. Lecteur égaré dans Simon comme dans un monde qui le déborde, le fascine. En hypnose et en apnée, souffle coupé pour une plongée dont on ne revient pas indemne. Mais à laquelle on revient sans cesse, inassouvi. Parce que cette phrase-monde recèle encore tant de choses inaperçues, lovées dans les ramures d'une syntaxe proliférante, d'une culture bousculée.

Ce monde présent comme une énigme, il ne voulait pas s'en tenir quitte, l'abandonnant à son " être-là " avec indifférence : au contraire son écriture s'acharnait à le comprendre, et ne cessait d'en creuser les obscurités, les incongruités, les surprises. Comprendre : tel est l'enjeu, mais au sens étymologique d'abord : tout prendre avec soi dans la nasse des mots qui entraînent ailleurs, avec leurs jeux de poupées gigognes où le lexique appelle à lui tous les sens possibles, et les fait rayonner. Claude Simon : une écriture des carrefours qui ne se satisfait pas d'un seul chemin, mais les explore tous. Et sans raison systématique, jamais un par un, mais tous de concert, fouillant de l'un à l'autre, faisant passerelle et retour, chemins de traverses et broussailles. Holzwege qu'un hasard ramène au port.

Ce monde comme une énigme dont il déchiffrait les signes, abandonnant plus d'une fois le hasard du déchiffrement au miroitement des signes, comme arrêté dans son élan pour mieux les contempler. Les décrire. Et nous arrêtant avec lui dans l'épiphanie de moments arrachés au cours du temps. Pour cela, il avait inventé le participe présent. Inventé, c'est-à-dire désenfoui, repris là où Faulkner l'avait laissé, et plus largement employé encore. Dans cette langue française où la forme progressive n'existe guère, il a ralenti les progressions. Maintenant les images dans leur suspens, décomposant les mouvements, immobilisant leur flux, comme dans Triptyque, ce poisson - était-ce une truite ? - immobile à remonter le courant. Remontant le flot, le flux d'un temps désentravé des mémoires. Une énigme dont il déchiffrait les signes cependant. $\mathrm{Ce}-$ pendant : pendant qu'il en décrivait les miroitements, intrigué de savoir ce qu'il y avait derrière tant d'ostentation, comme derrière le rideau de paon de La Route des Flandres. 
Ou, plus exactement, intrigué par son propre goût pour l'ostentation. Non pas pour la sienne, peu d'écrivains furent aussi discrets, aussi secrets même que Claude Simon. Non, mais pour le goût qu'il avait, lui, Simon, l'écrivain, pour l'ostentation des choses, celles qui se pressent aux sens, les sollicitent de manière si intense qu' elles mettent le reste du monde, les affaires auxquelles nous vaquons, les intrigues et les existences, comme entre parenthèses, le temps d'une phrase qui s'y porte, les enrobe, et se laisse, par elles, entraîner, si loin parfois que des choses mêmes elle a perdu le cours. Ce goût pour l'ostentation des choses, ce goût des parenthèses qui les capturent, l'œuvre semble depuis ses débuts être partie en quête de leurs motivations. Attachée, lorsqu'elle ne s'y laisse pas complètement absorber, à savoir pourquoi. Non pas seulement " comment est-ce ; comment était-ce?", non pas seulement " que savoir, comment savoir ? ", mais aussi - et sans le dire - "pourquoi vouloir ?" : connaître la raison de son désir.

Et ce n'est qu'à sa fin, au terme presque venu d'un si long et si riche parcours, que l'écrivain put mettre un nom sur son inextinguible fascination : " et à la fin, il dit : Mélancolie ». Mélancolie : pas la tristesse : l'avidité. Une avidité qui se porte au présent comme au passé : qui ne se satisfait pas de retenir ce qui est, mais cherche à restituer ce qui fut. De quelques cartes postales, rebâtir une histoire coloniale et matrimoniale ; de quelques lettres, dessiner un général d'Empire, un haras et des terres ; un père avec une montre et une balle tordue. Restitution: c'est peut-être, parmi d'autres, le legs de ce geste-là qui demeure le plus vif dans la littérature d'aujourd'hui, à laquelle Claude Simon a tant donné. Son souci de ce qui fut, non pas exactement tel que cela fut - comment le savoir, en effet, depuis cet aujourd'hui où l'on écrit ? - mais tel qu'aujourd'hui, avec les moyens du bord, grâce à ce fabuleux bricolage de l'écriture auquel il nous initie, on peut tenter de l'écrire.

Je crois symptomatique que nombre d'écrivains et d'artistes aient accepté notre invitation d'écrire à leur tour ce qu'ils retiennent des romans de Claude Simon, et que pour la plupart, ils l'aient fait ainsi : non par un message hâtif de témoignage et de sympathie, mais en prenant le temps d'écrire combien, à leur façon, ils demeu- 
rent pris dans l'entrelacs de l'œuvre. Dans sa phrase justement, le plus souvent : ils sont plusieurs à le dire - et comment ! Dans les questions qu'elle agite, aussi, et dans l'Histoire qu' elle traverse, dont les errements et la noirceur ne sont pas plus dissimulés que ne l'est le «monde fastueux, inépuisable». Ce livre témoigne : la littérature présente, sans doute, ne serait pas la même si les textes de Claude Simon n'en avaient fécondé l'inquiétude, lui refusant le repos des certitudes, lui offrant l'exigence d'écrire comme un enjeu où éthique et esthétique se rejoignent et se relancent.

Bien sûr je devrais, au nom de l'Association des Lecteurs de Claude Simon, les remercier, tous, écrivains et artistes, d'être là. D'avoir, chacun à sa façon et toutes générations confondues, répondu présent à notre appel. Mais ce serait déplacer les choses, car c'est ensemble que nous remercions ici Claude Simon d'une œuvre qui ne cesse de nous habiter, de nous hanter parfois. Et d'inspirer aussi à la littérature qui vient, comme les textes qui suivent le laissent entendre, quelques-unes de ses plus belles pages. On a dit, écrit, ici et là, qu'à l'ombre des grandes figures disparues, Simon, Beckett..., il pouvait être difficile d'écrire encore. Cet hommage et les contributions qu'il rassemble prouvent le contraire : un grand écrivain vivifie la création littéraire par les voies inexplorées qu'il ose, par les désirs qu'il suscite, par le souffle de ses livres : l'œuvre de Claude Simon continue de s'ouvrir dans nos mains.

Il avait vu la mort en face et l'avait tenue, non en respect, mais en suspens durant plus de soixante-cinq ans. Le temps d'écrire une œuvre qu'elle n'emporterait pas. Elle a fini par le prendre, au début de juillet 2005. Mais il avait eu le temps de consigner le monde et ses géographies disparates, les saisons et leurs infinies variations, l'Histoire aussi, ses meurtrissures comme son ironie. De les consigner sans les classer : dans leur vrac et leur chaos, leurs entremêlements. Son œuvre écrit la présence du monde comme son effacement permanent, ses fureurs et ses incertitudes. Il nous les laisse tels, pour jamais inachevés, aussi impénétrables qu'énigmatiques. Mais 
nous pouvons continuer de les lire, et de les relire, dans des livres inimitables, marqués au coin par le battement interrogateur de son nom, Claude Simon, tel que ses initiales le recouvrent, C.S. : " c'est; est-ce?" 